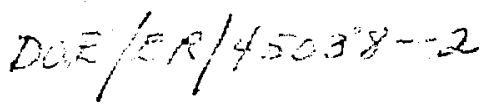 \\ $\operatorname{coN} F-840764--8$
}

\section{High Pressure X-Ray Absorption Studies of Phase Transitions}

J.M. Tranquada* and R. Ingalls

CONF-840764--8

Department of Physics, FM-15, University of Washington Seattle, WA 98195, USA

DE86 000548

\section{E.D. Crozier}

Department of Physics, Simon Fraser University

Burnaby, B.C. V5A 156, Canada

\section{Introduction}

The application of high pressure to a substance generally changes all of its properties to some degree. One of the most striking changes is a pressure-induced phase transition. Here we review how such phase changes reveal themselves in $x$-ray absorption spectra. The examples given are from our own work at SSRL, the experinental details having been previously reported [1]. The EXAFS-derived bond compression of a standard are used to determine the pressure.

\section{Pressure Dependence of the EXAFS}

We have previously reported the effects of pressure on the XANES of some materials undergoing phase transitions [2]. Although we shall aga in discuss such effects, it is our main purpose here to show the characteristic behavior of the EXAFS in such cases. We choose to write the EXAFS

- interference function $x(k)$ in terms of the cumulants $u(n)$ which characterize the various pair distribution functions $[3,4]$. For a given shell,

$$
x(k)=\frac{N}{k R^{2}} F(k) e^{-2 R / \lambda} e^{A} \sin B \text {. }
$$

- where the arguments $A$ and 8 are given by

$$
\begin{aligned}
& A=-2 v^{2} k^{2}+\frac{2}{3} v^{(4)} k^{4}+\ldots . \quad \text { and } \\
& B=2 k R+\phi(k)-\frac{4 a^{2} k}{R}\left(1+\frac{R}{\lambda}\right)-\frac{4}{3} v^{(3)} k^{3}+\ldots
\end{aligned}
$$

As pressure is applied, essentially all of the parameters in Eqs. (1 - 3) are expected to vary, with the structural parameters $N, R$, and $\sigma^{2}$ expected to exhibit the most significant changes. Using standard EXAFS analysis, the term

$$
\ln \left|\frac{N_{a} R_{b}^{2}}{N_{b} R_{a}{ }^{2}}\right|-\frac{2 \Delta R}{\lambda}-2 \Delta \sigma^{2} k^{2}+\frac{2}{3} \Delta v^{(4)} k^{4}+\ldots
$$

is extracted from the logarithm of the ratio of the amplitude of spectrum vs spectrum $b$. Similarly, the term

$\left.2 k i \Delta R-\frac{\Delta v^{2}}{\bar{R}}\left[1+\frac{\bar{R}}{\bar{\lambda}}\right)\right]-\frac{4}{3} \Delta \sigma{ }^{(3)} k^{3}+\cdots$

is obtained from the phase difference between two runs. The main point here is that there is a minor degres of coupling between fits to $\Delta R$ and $\Delta \sigma^{2}$, vs pressure. Also, because of anharmonicity, one generally must deal with a nonlinear fit in $k^{2}$ or $k$, respectively.

Present address, Brookhaven National Laboratory, Upton, NY 11973. 
The above type of analys is has been made for the Br k-edge spectra in $\mathrm{NaBr}$. Figure 1 shows the transforms of the data for three pressures, where one clearly sees the effects of compression and decreasing themal disorder. After a back transformation of the nearest neighibor peak, the va-ious parameters in Eqs. (4) and (5) may be determined. We have found it idvantagrous to first determine the room temperature atmospheric pressure curiulants through comparison with low temperature data, which is assumed to correspond to the harmionic case. The main result from the pressure experinents yields $\Delta R$ and $\Delta \sigma^{2}$ values which a spear to be nearly linearly relate.t. For $\mathrm{NaBr}$ we express this relation as $\left.\frac{\Delta \sigma^{2} / 0^{2}}{i R / R}\right|_{p=0} \simeq 12$. Interpreting these results in $t$ rms of an Einstein model would lead to a Grüneisen constant $\gamma_{E}=\frac{d \ell n \omega E}{d \ell n V}$ of about $1.56[4]$.

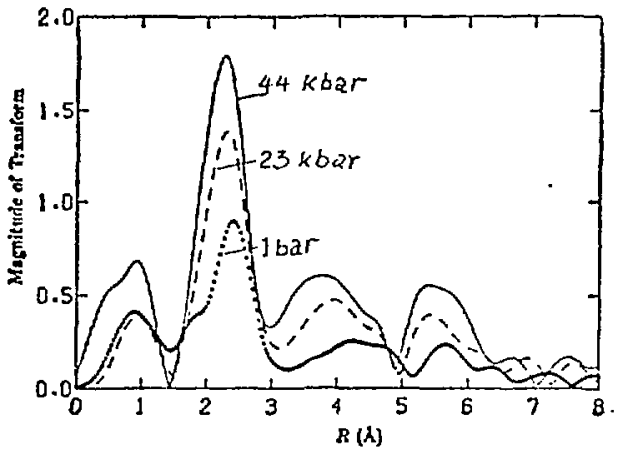

Fig. 1.

Fovicier transforms of $k^{3} a(k)$ for was

At atmospheric pressure $\mathrm{RbCl}$ has the same $B 1$ (rock-salt) structure as $\mathrm{NaBr}$. However, at about 5.2 [kbar] it transforms to the 32 (cesium chloride) structure. We have rollowed the EXAFS of RoCl through this phase transition and performed an analysis similar to that for liakr described above. The main results are shown in $\mathrm{Fig}$. 2. At low pressures $\left(\frac{i R}{R}=0\right)$ the data yield $\left.\frac{\Delta \sigma^{2} / \sigma^{2}}{\Delta R / R}\right|_{0}=16.7$, which would correspond to an Grüneisen paraneter of $\gamma_{E}=2.78$. This result appears to hold for both phases, that is, both the near neighbor distance and its $c^{2}$ decrease with piessure except at the $B]$ to $B 2$ transition wiere tiney incledse sligitily. The slight increase in bond length at the transition reflects the fact that the

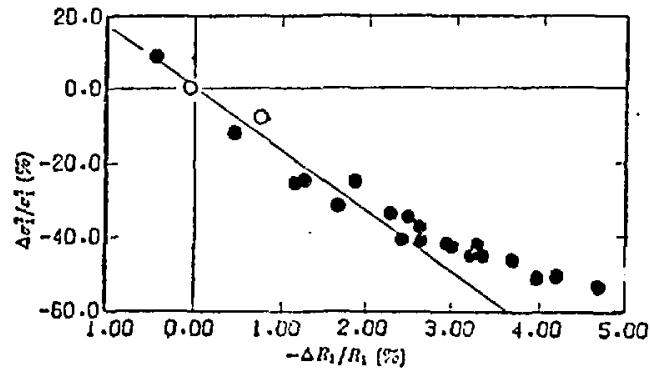

Fig.2. Change in $\alpha^{2}$ vs R. for nearest neighbor distance in RbCl. Open(closed) symbols represent the $B 1$ ( $B 2)$ phase 
coordination goes from 6-fold to 8-fold while the voluale itself decreases by approximately $14 \mathrm{~s}$. The accompanying increase in ${ }^{2}$ signifius a softening of the appropriate force constants. (The data is also consistent with $N$ increasing from 6 to 8 .)

He have carried out such an analysis in yet a more complex case, manely CuBr, which undergoes two phase transitions with pressure: a sharp transition from Cu\&r III to CuBr V (ziacblende to testragoml) al about 50 [kbar] and a sluggish transition from CuBr $V$ to $C u B r V I$ (BI structure) between 60 and 80 ikbarl. The data for both $K$ edges are consistes: with a nearest neighbor coordination of $N=4$ for the tetragonal phase, the cxact structure of which is not yet known, and it $=6$ for the $B l$ phase. Again, allalyzing the data in terms of $A \mathrm{C}^{2}$ vS $\Delta R$ yields a relation similar to Habr and $R B C l$ (Fig. 3). At each transition there appcars to be an increase in $B u^{2}$ and $\Delta R$ followed by a decrease as the pressure increases further. We remark here that ZnSe also transforms to a rock-salt phase at high bressure; a sini lar instability is reflected in the pressure dependence of 0 ' for the second siell. This is discussed further in another paper at this conference [5].

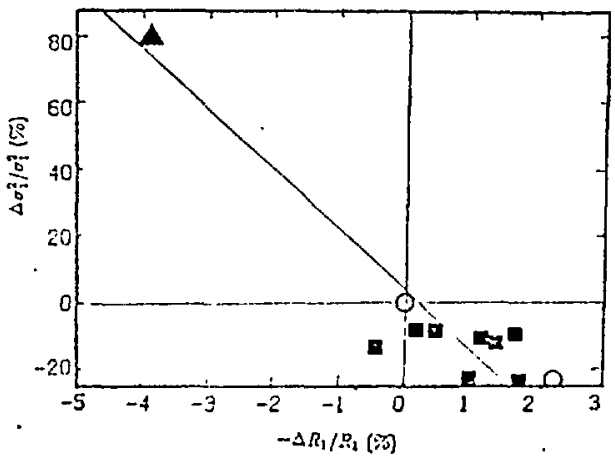

Fig. 3. Charge in $0^{2}$ vs $R$ for the nearest neighbor distance in Cubir III (O), Cubr V (a) and CuBr VI (i)

\section{Pressure Dependerice of the XANES}

Figures 4 and 5 show the changes in the XANES with the pressure-induced changes in crystal structure, for $\mathrm{RbCl}$ and $\mathrm{CuBr}$. A definitive detailed explanation of such behavior is not yet avaliable. We have also been studying the mixed-valent materials SmS and SmSe which undergo isostructural insulator-netal transitions with pressure. Figure 6 shows the XANES

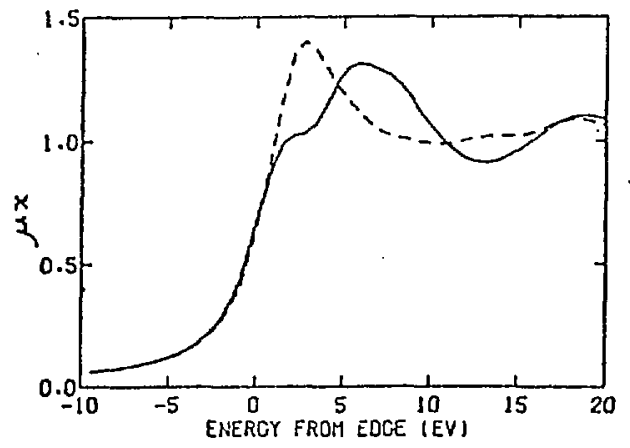

Fig. 4. Rb k-edge XANES in the B1 (solid line) and B2 (dashed line) phases of $\mathrm{RbCl}$ 


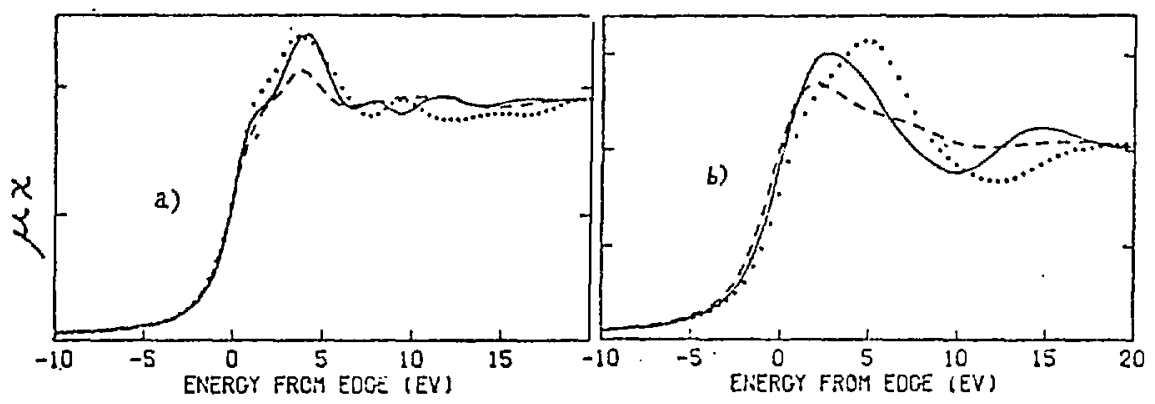

Fig. 5. a) Cu, and b) Br k-edge Xalics in the CuEr III (solid line), CuBr V (dashed line) and CuBr VI (dotted line) phases

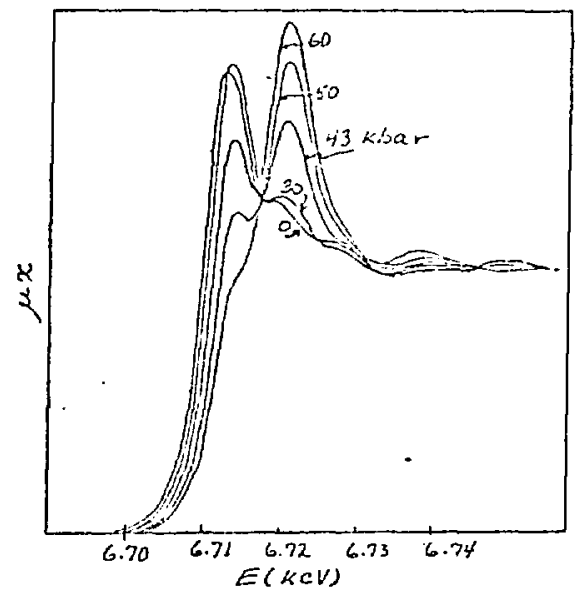

Fig. 6. Sili L 3 -edge XANES of SmSe at several pressures

behavior of the $\operatorname{Sin} L_{3}$ edge in SmSe, for example. Although, to our knowledge, these spectra have not been explained from first principles, they represent the change from the divalent $4 f^{6}$ to trivalent $4 f^{5}$ state, in which the photoexcitation threshold is roughly 7 ev higher, accompanied by a volume collapse.

We also find that if the fractional valence is determined from the relative areas under the two peaks, it does not follow $C R$ in a linear manner. That is after correcting for the usual compressibility, the collapse in $\Delta R$ occurs at a lowe: pressure than the valence change.

Similarly, and as reported by FRANK et a 1. [6] for SmS, we find that $\sigma^{2}$ for the nearest neighbor shell appears to increase and then decrease,going through the transition despite the large decrease in $\Delta R$. This would be in contiast to the usual Grineisen relationship described above for the other systems. It renains to be determined whether such a result is related to thermal disorder or some type of static structural disorder, possibly resulting from some type of inhomogeneity. 
We would like to express our thunks to uther menbers of our rosuarch groups, H. Alberding, R. Bauchspiess, B. llouser, R. Goen, A. Seary, P. Viren, and J. bin itmore. This work was supported in firt by the U.S. Iationis So ience Foundation, the Department of Encrgy, and by the llational sciences and Engineering Research Council of Canada.

1. R. Ingalls, E.D. Crozier, J.E. bhitirore, A.J. Seary, and J.H. Tranquada, J. Aipl. Phys. 51, 3158 (1980)

2. R. Ingalls, J.M. Tranquada, J.E. Whili..re and E.D. Crozier in EAAFS and Near Fdge Structure, A. Bianconi, L. Incoccia and S. Stifelich, eds. (Springer-Vertag, Berlin, 1983) 0. 153

3. J.M. Tranquada and R. Ingalls, Phys. PRe. is 23, 3520 (1983)

4. J.M. Tranquada, Ph.D. Thes is, Univ. of hashington, 1983 (umpublished)

j. J.H. Tranquada and R. Ingalls, this confurence.

б. K.H. Frank, G. Kaindl, J. Feldhaus, G. Wroman, W. Krone, G. Fa terlik, and $H$. Bach, in Valence Instabilities, P. Wachter and H. Boppart, eds. (North Holland, Ansterdan, 1982) p. 139 


\section{DISCLAIMER}

This report was prepared as an account of work sponsored by an agency of the United States Government. Neither the United States Government nor any agency thereof, nor any of their employees, makes any warranty, express or implied, or assumes any legal liability or responsibility for the accuracy, completeness, or usefulness of any information, apparatus, product, or process disclosed, or represents that its use would not infringe privately owned rights. Reference herein to any specific commercial product, process, or service by trade name, trademark, manufacturer, or otherwise does not necessarily constitute or imply its endorsement, recommendation, or favoring by the United States Government or any agency thereof. The views and opinions of authors expressed herein do not necessarily state or reflect those of the United States Government or any agency thereof. 